\title{
Neurological Diseases and Relation of TRP Channels
}

\author{
Betül Yazğan ${ }^{1}$ and Yener Yazğan ${ }^{2 *}$ \\ ${ }^{1}$ Department of Physiology, Medical Faculty, Adlyaman University, Turkey \\ ${ }^{2}$ Department of Neuroscience, Medical Faculty, Suleyman Demirel University, Turkey
}

Received: 啙July 17, 2018; Published: 眥July 25, 2018

*Corresponding author: Yener Yazğan, Department of Neuroscience, Medical Faculty, Suleyman Demirel University, Isparta, Turkey

\begin{abstract}
Is currently used as a comprehensive definition covering "brain disorders", neurological diseases and mental disorders. While schizophrenia, depression, panic disorder, drug addiction and insomnia are referred to as "mental disorders", Epilepsy, Alzheimer, Parkinson, Huntington's disease (HD) and multiple sclerosis are considered as "neurological disorders". Current therapies of these very common diseases require continuous drug use and at the same time cause many different side effects. Therefore preclinical and clinical investigations for new treatment approaches are on the rise. Especially the identification of the molecular basis of these diseases is the focus of researches.
\end{abstract}

Examination of transient receptor potential (TRP) channels is at an early stage in the investigation of the molecular principle of these diseases, but clear results regarding the efficacy of substances activating or inhibiting these channels have not been obtained. Some diseases have been based on mutations of TRP channels. However, only a few TRP channelopathies, have been conclusively identified so far [1]. Investigation of TRP channels in psychiatric disorders will contribute to a better understanding of the etiology of psychiatric disorders and the development of new pharmacological treatments.

Abbreviations: TRP: Transient Receptor Potential; GPCR: G Protein Coupled Receptor; TRPV1: TRP Vanilloid 1; CGRP: Calcitonin Gene Related Peptide; CNS: Central Nervous System; SOC: Store Operated Calcium Channels; BDNF: Brain Derived Neurotrophic Factor; Mwk: Moon Walker Mouse; BD-I: Bipolar Disorder Type I; ALS-G: Guamanian Amyotrophic Lateral Sclerosis

\section{TRP Superfamily}

TRP channels are plasma membranes, non-selective cationic channels, and have been demonstrated to play a role in the regulation of cellular $\mathrm{Ca}^{2+}$ influx [2,3]. These channels have been shown to be part of the TRP family of store-operated channels. TRP channel is provided by both the direct action on the channel of a multiplicity of exogenous and endogenous physicochemical stimuli, and changes in the intracellular mechanism, including activation of G-protein coupled receptor (GPCR) or tyrosine kinase receptor [4]. TRP cation channels act as cellular sensors, which play crucial roles in most of the physiological processes. Based on the sequence homology, mammalian TRP channels be separated, six subfamilies: TRP canonical (TRPC; TRPC1-7), TRP vanilloid (TRPV; TRPV1-6), TRP melastatin (TRPM; TRPM1-8), TRP polycystin (TRPP; TRPP2, TRPP3, TRPP5), TRP mucolipin (TRPML; TRPML1-3), and TRP ankyrin (TRPA; TRPA1). All TRP channels are membrane proteins with six putative transmembrane segments (S1-S6) and a cationpermeable pore region between S5 and S6. The cytoplasmic amino (N) and carboxy (C) termini are variable in length and contain different domains (e.g., ankyrin repeats, coiled-coil, calmodulin binding sites, etc.).

The domain composition can vary significantly between subfamily members. Functional TRP channels are either homoor heterotetramers assembled from identical or similar TRP subunits [2]. Since their recognition, research on the role of TRP channels in health and disease has increased steadily. There is very strong evidence that they are important in neuronal stimulation, nociceptive responses, allodynia and hyperalgesia [5]. 


\section{TRP Channels in Brain Disorders}

\section{TRPV1 and TRPA1}

Significant evidence has been obtained that TRPV1 antagonists have anxiolytic activity in preclinical studies, but the antidepressant effect is not clear [6]. There is no direct evidence that TRP channels play a role in schizophrenia. However, the fact that TRPV1 channels play a role in central dopaminergic and cannabinoid mechanisms may suggests the potential role of these channels in schizophrenia [5]. CGRP release from trigeminal vasculature neuron network and neurogenic inflammatory response are currently accepted mechanisms for migraine attack pathophysiology. In particular, the TRP vanilloid 1 (TRPV1) and the TRP ankyrin 1 (TRPA1) are expressed in nociceptive neurons, which also express the sensory neuropeptides, tachykinins, and calcitonin gene-related peptide (CGRP), which mediate neurogenic inflammatory responses. This evidence suggests that these channels may be an important therapeutic goal in migraine treatment [7].

\section{TRPC Subfamily}

TRPC channels are associated with neuronal development and basic synaptic mechanisms. TRPC1 is the most widely distributed member of the TRPC subfamily in the central nervous system (CNS). TRPC1 is highly expressed in the hippocampus, amygdala, cerebellum [8], substantia nigra [9] and inferior colliculus [10]. TRPC5 is co-localized with TRPC1 in the rat substantia nigra [11].

TRPC1 participates in important neuronal processes related to synaptic transmission and plasticity [12]. In some brain disorders, TRPC1 plays a noteworthy role. Epileptiform burst firing, induced by group I mGluR agonists, reduces in TRPC1 knock-out mice. Intracellular calcium imbalance, which are related to store operated calcium channels (SOC), are also involved in neurological disorders such as Alzheimer's, Huntington's disease and epilepsy. Neurotoxicity following epileptic seizure activity is frequently observed. TRPC4 and TRPC1 were found to mediate neuronal cell death in response to seizures in the lateral septal and hippocampal neurons [13]. These investigators have shown that the TRPC1 / TRPC4 heteromultimeric complex is activated by metabotropic glutamate receptors, as well as by neuronal firing which leads to epileptiform burst in the lateral septum [14].

Selvaraj et al. 2012, showed that TRPC1 may be a possible target for Parkinson's disease [15]. They proved reduction in the number of dopaminergic neurons in the substantia nigra in TRPC1-/- mice. Also, Consistently, brain lysates of patients with Parkinson's disease demonstrated decreased levels of TRPC1. Further studies are currently underway to evaluate possible changes in TRPC receptors in substantia nigra after experimental Parkinson's disease [16].

TRPC3 channels were suggested to mediate brain-derived neurotrophic factor (BDNF)-dependent dendritic remodelling in the hippocampus [17]. In a transgenic mouse model, the relationship between TRPC3 and the development of a recessive neuromotor disease has been investigated. Transgenic model is TRPC3 transcription and TRPC3 channel expression blockade. Atrophy and paralysis were observed in the back legs of transgenic mice [18].

In another study, the relationship between TRPC3 and cerebellar ataxia was investigated in a moon walker mouse (Mwk), a model for cerebellar ataxia. TRPC3 is highly expressed in Purkinje cells during dendritogenesis. Growth and differentiation of purkinje cell dendrites are dramatically deteriorating in Mwk mouse. For this reason, it may be suggested that the pathogenesis of cerebellar ataxia may involve upregulation of TRPC3 function [19].

\section{TRPM Subfamily}

TRPM2 and TRPM7 have vital precautions for cell viability. They are important regulators in many neurodegenerative disease processes, including Alzheimer's disease and amyotrophic lateral sclerosis, due to their oxidative stress (especially TRPM2) perceptions and their direct involvement in neuronal cell death pathways [20].

Guamanian amyotrophic lateral sclerosis (ALS-G) and parkinsonism dementia (PD-G or parkinsonism dementia complex, PDC), which are caused by mutations in TRPM2 and TRPM7 genes, two neurodegenerative disorders are with relatively high incidence in Guam of the Pacific Islands [21]. One of the putative susceptibility locus of bipolar disorder type I (BD-I) is associated with chromosomal regions encoding TRPM2. SNPs in the promoter region of TRPM2 are also significantly associated with bipolar disorder type II (BD-II), suggesting that TRPM2 polymorphisms contribute to both types of BD [22].

\section{Results}

Further analysis of the neuroanatomical distribution of the subtypes of TRP channels and the role of these channels in physiological processes in the brain will contribute to an understanding of the molecular basis of neurological diseases. It is also useful to identify in detail the new etiologic and endogenous factors affecting these channels so that TRP channels can be recommended as a new therapeutic target in neurological diseases.

\section{References}

1. Nilius B, Prenen J, Owsianik G (2011) Irritating channels: the case of TRPA1. J Physiol 589(7): 1543-1549.

2. Yazğan Y, Nazıroğlu M (2017) Ovariectomy-Induced Mitochondrial Oxidative Stress, Apoptosis, and Calcium Ion Influx Through TRPA1, TRPM2, and TRPV1 Are Prevented by $17 \beta$-Estradiol, Tamoxifen, and Raloxifene in the Hippocampus and Dorsal Root Ganglion of Rats. Mol Neurobiol 54(10): 7620-7638.

3. Nilius B, Owsianik G (2011) The transient receptor potential family of ion channels. Genome Biol 12(3): 218.

4. Benemei S, De Cesaris F, Fusi C, Rossi E, Lupi C, Geppetti P (2013) TRPA1 and other TRP channels in migraine. J Headache Pain 14: 71.

5. Mobasheri A, Barrett Jolley R (2011) Transient receptor potential channels: emerging roles in health and disease. Vet J 187(2): 145-146. 
6. Socała K, Wlaź P (2016) Evaluation of the antidepressant- and anxiolyticlike activity of $\alpha$-spinasterol, a plant derivative with TRPV1 antagonistic effects, in mice. Behav Brain Res 303: 19-25.

7. Utsumi D, Matsumoto K, Tsukahara T, Amagase K, Tominaga M, et al (2018) Transient receptor potential vanilloid 1 and transient receptor potential ankyrin 1 contribute to the progression of colonic inflammation in dextran sulfate sodium-induced colitis in mice: Links to calcitonin gene-related peptide and substance P. J Pharmacol Sci 136(3): 121-132.

8. Martinez Galan JR, Verdejo A, Caminos E (2018) TRPC1 Channels Are Expressed in Pyramidal Neurons and in a Subset of Somatostatin Interneurons in the Rat Neocortex. Front Neuroanat 12: 15.

9. Martorana A, Giampà C, DeMarch Z, Viscomi MT, Patassini S, et al. (2006) Distribution of TRPC1 receptors in dendrites of rat substantia nigra: a confocal and electron microscopy study. Eur J Neurosci 24(3):732-738.

10. Valero ML, Caminos E, Juiz JM, Martinez Galan JR (2015) TRPC1 and metabotropic glutamate receptor expression in rat auditory midbrain neurons. Neurosci Res 93(6): 964-972.

11. CE Gee, P Benquet, U Gerber (2003) Group I metabotropic glutamate receptors activate a calcium-sensitive transient receptor potential-like conductance in rat hippocampus. J Physiol 546(Pt 3): 655-664.

12. Bröker Lai J, Kollewe A, Schindeldecker B, Pohle J ,Nguyen Chi V, et al. (2017) Heteromeric channels formed by TRPC1, TRPC4 and TRPC5 define hippocampal synaptic transmission and working memory. EMBO J 36(18): 2770-2789.

13. Phelan KD, Shwe UT, Abramowitz J, Wu H, Rhee SW, et al. (2013) Canonical transient receptor channel 5 (TRPC5) and TRPC1/4 contribute to seizure and excitotoxicity by distinct cellular mechanisms. Mol Pharmacol 83(2): 429-438.

14. Phelan KD, Mock MM, Kretz O, Shwe UT, Kozhemyakin M, et al. (2012) Heteromeric canonical transient receptor potential 1 and 4 channels play a critical role in epileptiform burst firing and seizure-induced neurodegeneration. Mol Pharmacol 81(3): 384-392.

15. Selvaraj S, Sun Y, Watt JA, Wang S, Lei S, Birnbaumer L, Singh BB (2012) Neurotoxin-induced ER stress in mouse dopaminergic neurons involves downregulation of TRPC1 and inhibition of AKT/mTOR signaling. J Clin Invest 122(4): 1354-1367.

16. Alessandro M, Carmela G, Zena D, Maria TV, Stefano P, et al. (2006) FuscoDistribution of TRPC1 receptors in dendrites of rat substantia nigra: a confocal and electron microscopy study. Eur J Neurosci 24(3): 732-738.

17. Hang P, Zhao J, Cai B, Tian S, Huang W, et al. (2015) Brainderived neurotrophic factor regulates TRPC3/6 channels and protects against myocardial infarction in rodents. Int J Biol Sci 11(5): 536-545.

18. Eder P, Groschner K (2008) TRPC3/6/7: Topical aspects of biophysics and pathophysiology. 2(2): 94-99.

19. Becker EBE, Oliver PL, Glitsch MD, Banks GT, Achillic F, et al. (2009) A point mutation in TRPC3 causes abnormal Purkinje cell development and cerebellar ataxia in moonwalker mice. Proc Natl Acad Sci USA 106(16): 6706-6711.

20. Hermosura MC, Garruto RM (2007) TRPM7 and TRPM2-Candidate susceptibility genes for Western Pacific ALS and PD? Biochim Biophys Acta 1772(8): 822-835.

21. Nilius B, OwsianikG (2010) Transient receptor potential channelopathies. Pflugers Arch 460(2): 437-450.

22. Xu C, Macciardi F, Li PP, Yoon IS, Cooke RG, et al. (2006) Association of the putative susceptibility gene, transient receptor potential protein melastatin type 2, with bipolar disorder. Am J Med Genet B Neuropsychiatr Genet 141: 36-43.

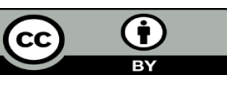

This work is licensed under Creative Commons Attribution 4.0 License

To Submit Your Article Click Here:

Submit Article

DOI: 10.32474/OJNBD.2018.01.000120

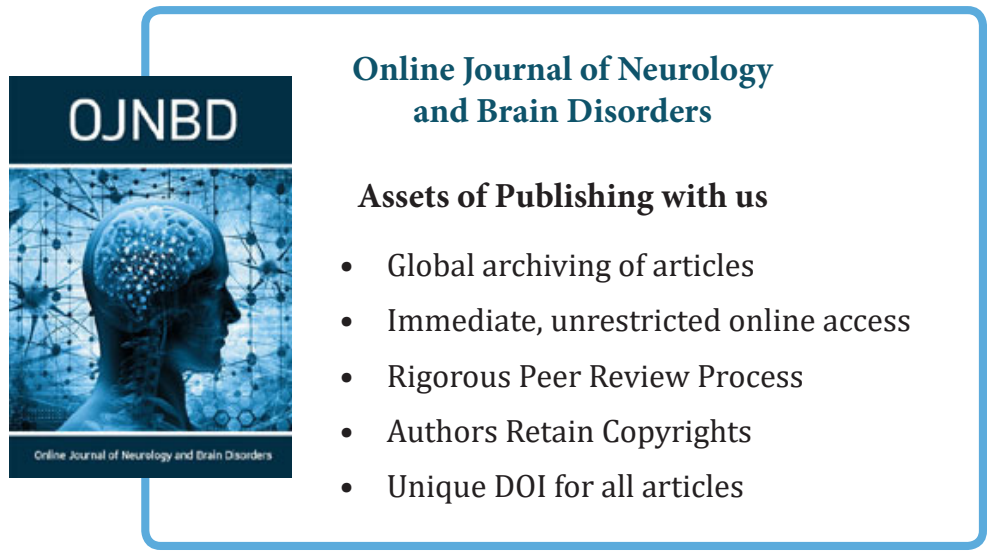

